\title{
Does CT scanning predict the likelihood of a positive transbronchial biopsy in sarcoidosis?
}

\author{
S de Boer, ${ }^{1}$ D G Milne, ${ }^{2}$ I Zeng, ${ }^{1}$ M L Wilsher ${ }^{1}$
}

\begin{abstract}
- Table 5 is published online only at http://thorax.bmj.com/ content/vol64/issue5

${ }^{1}$ Green Lane Respiratory Services, Auckland City Hospital, Auckland District Health Board, Auckland, New Zealand;

${ }^{2}$ Department of Radiology, Auckland City Hospital, Auckland District Health Board, Auckland, New Zealand
\end{abstract}

Correspondence to: Dr S de Boer, Green Lane Respiratory Services, Auckland City Hospital, Private Bag 92 024, Auckland 01, New Zealand; sdeboer@adhb.govt.nz

Received 20 July 2008 Accepted 12 January 2009 Published Online First 12 February 2009
ABSTRACT

Background: Transbronchial lung biopsy (TBB) has a variable diagnostic yield in sarcoidosis. It was hypothesised that the extent and pattern of parenchymal disease on CT scanning would predict the likelihood of a positive TBB result.

Methods: Patients with sarcoidosis $(n=77)$ were included if they had undergone a CT scan within 6 weeks of TBB. Ethnicity, symptoms, pulmonary function and site and results of TBB and bronchoalveolar lavage (BAL) were recorded. CT scans were scored quantitatively for patterns of parenchymal disease (nodular, reticular, consolidation and ground glass) on a lobar basis.

Results: 39 patients (50.6\%) had a positive TBB. Symptoms, ethnicity, treatment, lung volumes and chest radiographic stage were not predictors of a positive biopsy. Female gender, reduced percentage predicted carbon monoxide transfer factor and a higher percentage of lymphocytes in the BAL fluid were associated with a positive biopsy, as were higher total lung score, reticular pattern and ground-glass opacity. The associations were more significant for the total lobar score and the lobar ground-glass score of the lobe biopsied. On multivariate analysis gender, percentage of lymphocytes in the BAL fluid and total lung score were independent predictors of a positive TBB.

Conclusion: The total extent of parenchymal disease on the CT scan in addition to the pattern and lobar distribution predicts the likelihood of a positive TBB at bronchoscopy.

The diagnosis of sarcoidosis is based on a compatible clinical and radiological presentation and histological evidence of non-caseating granulomas with negative mycobacterial and fungal cultures. ${ }^{1}$ Over $90 \%$ of patients with sarcoidosis present with pulmonary involvement and bronchoscopy with transbronchial biopsy (TBB) is a convenient and safe method of securing a histological diagnosis. However, the diagnostic yield from TBB is variable ranging from $44 \%$ to $>90 \%$. $^{2-5}$

Efforts have been made to identify factors which are associated with obtaining a positive TBB. Sampling the lobe with the greatest parenchymal involvement on chest radiography, sampling more than one site and increasing the number of biopsies taken (up to perhaps 10 in stage $0 / 1$ disease) are associated with an increased yield. ${ }^{267}$ Neither symptoms nor abnormalities of pulmonary function are independent predictors of biopsy result once radiographic staging is removed. ${ }^{3}$ With advancing chest radiographic stage of disease the yield at TBB appears to increase, but no studies have specifically addressed the correlation between CT findings and TBB yield. We hypothesised that the extent, distribution and pattern of parenchymal disease on the CT scan would predict the likelihood of a positive TBB.

\section{METHODS}

Patients with a diagnosis of sarcoidosis undergoing bronchoscopy and TBB at Green Lane and Auckland City Hospitals, with a CT scan within 6 weeks, were identified retrospectively from bronchoscopy records. Ninety-seven potential subjects were identified, but 20 were excluded owing to incomplete clinical records or missing CT scans.

Demographic data, pulmonary function results and the site and results of bronchoalveolar lavage (BAL) and TBB were recorded from clinical notes. European Community Coal and Steel (ECCS) predicted values were used for pulmonary function data and results expressed as a percentage of the mean predicted values. A positive TBB was defined as a non-caseating granuloma in the absence of positive tissue stain for organisms.

The CT scans were retrospectively assessed in random order by an experienced observer (DM) without knowledge of the bronchoscopy result or lung function tests. CT patterns quantified were: (1) nodules up to $8 \mathrm{~mm}$ in diameter; (2) a reticular pattern (ie, interlacing linear opacities including thickened interlobular septa) or honeycombing; (3) ground glass opacification; and (4) consolidation. The extent of individual CT patterns was estimated in each lobe as follows: grade $1, \leqslant 25 \%$ of the lobe; grade 2, 26-50\% of the lobe; grade 3, $51 \%-75 \%$ of the lobe; grade $4,>75 \%$ of the lobe. The total extent of each pattern was determined by adding the scores in the six lobes, providing a 25 -point scale (0-24). The total extent of parenchymal disease (total lung score) was determined by adding the total extent of each pattern.

\section{Statistical analysis}

Continuous demographic and clinical variables are reported as mean (SD) values and BAL and CT score variables are reported as median (interquartile range). A $\chi^{2}$ test was applied to compare categorical variables between positive and negative biopsy groups, and the Fisher exact test was used where appropriate. The Mann-Whitney U test was used to compare CT patterns between positive and negative biopsy groups. Logistic regression was used to identify significant predictors for positive biopsy outcome. Independent predictors with $p<0.15$ in univariate analysis were further assessed by multiple logistic regression. Models with different combinations of significant independent predictors were investigated and the final models were selected based on adjusted $p$ value, maximised 
Table 1 Demographic, spirometric and BAL data from positive and negative TBB specimens

\begin{tabular}{|c|c|c|c|}
\hline & $\begin{array}{l}\text { Positive biopsy } \\
(\mathrm{n}=39)\end{array}$ & $\begin{array}{l}\text { Negative biopsy } \\
(\mathrm{n}=38)\end{array}$ & p Value \\
\hline Mean (SD) age (years) & $45(13)$ & $39(12)$ & 0.05 \\
\hline Female gender, n (\%) & $26(66.7)$ & $12(31.6)$ & 0.003 \\
\hline Ever smoked, n (\%) & $13(33.3)$ & $21(55.2)$ & 0.05 \\
\hline $\mathrm{FEV}_{1}(\% \text { predicted })^{*}$ & $88.4(22.6)$ & $91.4(15.5)$ & 0.60 \\
\hline FVC (\% predicted) ${ }^{*}$ & $87.6(18.9)$ & $93.8(12.0)$ & 0.24 \\
\hline TLCo $(\%$ predicted $) \dagger$ & $75.5(19.0)$ & $84.5(14.7)$ & 0.04 \\
\hline BAL \% lymphocytes: & $29.4(10.9,40.5)$ & $12.3(7.3,22.8)$ & 0.05 \\
\hline BAL \% neutrophils & $0.7(0.4,2.1)$ & $1.6(0.9,3.1)$ & 0.02 \\
\hline BAL \% eosinophils: & $0.5(0.2,1.2)$ & $0.3(0.1,0.9)$ & 0.24 \\
\hline BAL \% macrophages: & $60(53,72)$ & $79(57,86)$ & 0.03 \\
\hline
\end{tabular}

Demographic and lung function data presented as mean (SD). BAL data presented as median (interquartile range).

${ }^{*} \mathrm{FEV} / / \mathrm{FVC}$ data available for 75 patients. $\dagger$ TLCo data available for 66 patients. $\$ \mathrm{BAL}$ data available for 59 patients.

$\mathrm{BAL}$, bronchoalveolar lavage; $\mathrm{FEV}_{1}$, forced expiratory volume in $1 \mathrm{~s}$; FVC, forced vital capacity; TBB, transbronchial biopsy; TLCO, carbon monoxide transfer factor.

area under the receiver-operator curve (AUC) and low Akaike's information criterion (AIC).

\section{RESULTS}

Seventy-seven subjects $(50.6 \%$ male) were included of mean (SD) age 42.4 (12.7) years and ethnicity: European (76.6\%), Polynesian (10.4\%), Indian (9.1\%). The chest radiographic stage was as follows: stage $0,2.6 \%$; stage $1,38.9 \%$; stage $2,19.5 \%$; stage $3,28.6 \%$; stage $4,7.8 \%$; unknown, $2.6 \%$. Cough was the most common symptom $(\mathrm{n}=37)$ followed by dyspnoea $(\mathrm{n}=25)$ and wheeze $(\mathrm{n}=7)$. Twelve patients had no respiratory symptoms. Lung function was well preserved: mean forced expiratory volume in $1 \mathrm{~s}\left(\mathrm{FEV}_{1}\right) 89.9 \%$ predicted; forced vital capacity (FVC) $90.6 \%$ predicted; and carbon monoxide transfer factor (TLCO) $79.7 \%$ predicted. Eleven patients (14.3\%) had extrapulmonary manifestations of sarcoidosis at the time of bronchoscopy (six hypercalcaemia, three uveitis, one skin and one liver). Most patients (89.6\%) were receiving no treatment for sarcoidosis at the time of bronchoscopy.

TBB yielded a positive biopsy in 39 patients (50.6\%). Of the 38 patients with a negative TBB, histological diagnosis was subsequently obtained in 13 which resulted in $67 \%$ of the total cohort having histological confirmation of the diagnosis. Of the remaining 25 patients, the diagnosis was based on compatible clinical and radiological (CT) criteria alone. Pneumothorax as a complication of TBB was reported in two patients and bleeding $>20 \mathrm{ml}$ in one patient.

Fifty-nine patients had a BAL at the time of TBB. The biopsy was taken from the same lobe as the BAL in 23 patients. Five patients had endobronchial biopsy (EBB) in addition to TBB; in 1 of $5 \mathrm{EBB}$ positive specimens, TBB was negative; in 2 of $5 \mathrm{EBB}$ positive specimens, $\mathrm{TBB}$ was positive; and in 2 of $5 \mathrm{EBB}$ negative specimens, TBB was positive. One patient had a transbronchial

Table 2 Comparison between patients with and without parenchymal disease on the chest radiograph

\begin{tabular}{lllc}
\hline & $\begin{array}{l}\text { Chest radiograph } \\
\text { stage 0/1 } \\
(\mathbf{n = 3 2 )}\end{array}$ & $\begin{array}{l}\text { Chest radiograph } \\
\text { stage 2/3/4 } \\
(\mathbf{n = ~ 4 3 )}\end{array}$ & p Value \\
\hline $\begin{array}{l}\text { Positive biopsy, No (\%) } \\
\text { Total CT score, median }\end{array}$ & $14(43)$ & $24(56)$ & 0.3 \\
(IOR) & $5(3,6)$ & $15(9,20)$ & $<0.001$ \\
\end{tabular}

needle aspiration (TBNA) of a lymph node in addition to TBB with a positive result in the former test only.

Ethnicity and presenting symptoms were not significantly different between those with positive or negative TBB. Positive TBB was associated with increased age $(p=0.05)$, non-smokers $(p=0.05)$ and female gender $(p<0.01$, table 1$) . F V_{1}$ percentage predicted and FVC percentage predicted were not associated with positive biopsy but reduced TLCO percentage predicted was $(p=0.04)$. Patients with a positive TBB had higher percentage of lymphocytes $(p=0.05)$ and a lower percentage of neutrophils $(p=0.02)$ and macrophages $(p=0.03)$ in the BAL fluid.

There was no significant difference in chest radiography stage between those with positive or negative TBB, and specifically no difference between those with parenchymal disease on the chest radiograph (stage 2/3/4) and those without (stage 0/1) (table 2). Only 1 of the 32 patients with chest radiography stage $0 / 1$ had no parenchymal disease scored on the CT scan, however the median CT score of parenchymal disease was significantly higher in patients with chest radiography stage $2 / 3 / 4(p<0.001)$.

Increased total lung score (sum of patterns of parenchymal disease) was significantly associated with a positive TBB $(p=0.02$, table 3$)$. Reticular $(p=0.01)$ and ground-glass patterns $(p=0.04)$ were significantly predictive of a positive TBB.

Ten patients had a TBB specimen taken from more than one lobe, with the documented results not differentiating which biopsy site had yielded the definitive result. In the remaining 67 patients, the total lobar score (the extent of disease scored on the CT scan within the lobe that was biopsied) was more significantly associated with a positive result $(p=0.01$, table 4$)$ than the total lung score. Reticular $(p=0.04)$ and ground-glass patterns $(p=0.03)$ of disease within the biopsied lobe were also predictive of a positive TBB.

On multivariate analysis, gender, total lung score and the percentage of lymphocytes in the BAL fluid were the strongest predictors of a positive TBB (see table 5 in the online supplement).

\section{DISCUSSION}

In accordance with published guidelines, a tissue diagnosis of sarcoidosis is usually recommended unless the patient has presented with Lofgren's syndrome or with typical HRCT features in the context of compatible clinical features. ${ }^{18} \mathrm{TBB}$ is a generally well tolerated day case procedure that is associated with a low complication rate but, as many patients have no symptoms at the time of presentation, any invasive procedure must be justified. The diagnostic yield of TBB in published studies varies from $44 \%$ to $90 \%,^{2-5}$ generally higher with increasing chest radiographic stage of disease. This study, perhaps surprisingly, did not find a significant difference between chest radiography stage and result of biopsy. However, it does show that both the extent of parenchymal disease and the distribution on the CT scan relative to the biopsy site can predict the likelihood of a positive TBB, with reticular and ground-glass patterns particularly associated with a positive biopsy result. Previous studies that have commented on CT appearances in relation to TBB yield have made little reference to the extent and distribution of parenchymal disease, albeit noting that parenchymal disease is often evident on the CT scan in those with stage $0-1$ chest radiographs. ${ }^{90}$

\section{Relationship between chest radiography and TBB yield}

In this study $40 \%$ of the patients had stage $0 / 1$ chest radiographs yet all but one had evidence of parenchymal disease on the CT 
Table 3 Extent and pattern of disease on CT scan in positive and negative transbronchial biopsies

\begin{tabular}{lcll}
\hline & $\begin{array}{l}\text { Positive biopsy } \\
(\mathbf{n}=\mathbf{3 9})\end{array}$ & $\begin{array}{l}\text { Negative biopsy } \\
(\mathbf{n}=\mathbf{3 8})\end{array}$ & $\mathbf{p ~ V a l u \mathbf { * } ^ { * }}$ \\
\hline Total lung score & $11(6,20)$ & $5.5(4,13)$ & 0.02 \\
Total reticular score & $2(0,5)$ & $0.5(0,3)$ & 0.01 \\
Total nodular score & $6(2,11)$ & $4(3,6)$ & 0.08 \\
Total consolidation score & $0(0,1)$ & $0(0,3)$ & 0.34 \\
Total ground-glass score & $0(0,3)$ & $0(0,0)$ & 0.04 \\
\hline
\end{tabular}

Values are median (IQR).

*Mann-Whitney U test.

scan. There was no statistical difference in TBB yield between those with and those without parenchymal disease on chest radiography, despite higher CT scores in those with stage 2/3/4 chest radiographs. In stage $0 / 1$ disease, nodules and reticular changes were the most prominent parenchymal patterns mirroring the total cohort. The CT scores in this group are, however, too low to detect any clear lobar distribution. Based on our findings, the positive predictive value (PPV) for chest radiography stage $2 / 3 / 4$ is $56 \%$, whereas the PPV for CT ranged from $61 \%$ to $70 \%$ depending on the cut-off point. Despite the limitations of these calculations (selection bias of population in this study and impracticality of formally scoring CT scans in a clinical setting), these data support the use of CT scanning to predict the likelihood of a positive TBB over chest radiography.

\section{Relationship between CT patterns and TBB yield}

Sarcoidosis is associated with typical CT appearances of nodules (micro and macro), irregularly thickened bronchovascular bundles and interlobular septae, ground-glass opacity, consolidation with air bronchograms, fibrosis and honeycombing. ${ }^{11} 12$ In this study a reticular pattern significantly correlated with a positive TBB result. Although this pattern is often associated with fibrosis, it also includes thickened peribronchovascular interstitium and interlobular septae. Pathological studies have shown that granulomas with or without perigranulomatous inflammation form within connective tissue sheaths around pulmonary vessels and airways, and within interlobular lymphatics or veins respectively. ${ }^{13}$ It is therefore not surprising that biopsy specimens from these areas yield granulomas. Although a ground-glass appearance on the CT scan was originally thought to represent an alveolitis ${ }^{14}$ and may do in certain cases, it has also been shown to be due to granulomas in the intralobular septae, peribronchovascluar space and around small vessels and airways. ${ }^{13}$ Ground-glass density had a significant association with a positive TBB result, despite most patients in this cohort not having this pattern of disease and, when present, having upper lobe predominance. This probably reflects the fact that the very small granulomas responsible for this disease pattern are beyond the resolution of current CT scans and are more widespread than indicated radiologically. Although nodules are generally the most common parenchymal abnormality seen on CT scans in sarcoidosis, their distribution is patchy and the failure to demonstrate an association between this radiological pattern and a positive $\mathrm{TBB}$ result probably reflects sampling error.

\section{Relationship between lobar CT score and TBB yield}

Previous studies have shown that a positive TBB result is more likely from those lobes with greatest involvement on the chest radiograph. ${ }^{6}$ Data from this study are in keeping with those findings in that the extent of parenchymal disease on the CT scan
Table 4 Extent and pattern of disease on the CT scan within the lobe from which the biopsy was taken

\begin{tabular}{llll}
\hline & $\begin{array}{l}\text { Positive biopsy } \\
(\mathbf{n}=35)\end{array}$ & $\begin{array}{l}\text { Negative biopsy } \\
(\mathbf{n}=\mathbf{3 2})\end{array}$ & $\mathbf{p ~ V a l u { } ^ { * }}$ \\
\hline Total lobar score & $3(1,4)$ & $1(1,2)$ & 0.01 \\
Lobar reticular score & $1(0,1)$ & $0(0,1)$ & 0.04 \\
Lobar nodular score & $1(1,2)$ & $1(1,1)$ & 0.06 \\
Lobar consolidation score & $0(0,0.5)$ & $0(0,0.25)$ & 0.96 \\
Lobar ground-glass score & $0(0,1)$ & $0(0,0)$ & 0.03 \\
\hline
\end{tabular}

Values are median (IOR).

*Mann-Whitney U test.

in the lobe sampled is more significantly associated with a positive biopsy than the total extent of disease and, just as for the whole lung, a reticular and/or ground-glass pattern in the lobe sampled is associated with a positive TBB. The majority of TBB specimens in this study were taken from the right lower lobe, and it is not possible from retrospective documentation to know if this was due to that lobe being assessed as having the greatest parenchymal disease by the attending physician or because of ease of access. The yield of TBB in this study was increased to $67 \%$ in patients who had TBB specimens taken from the right lower lobe and had that lobe scored as the most diseased (ie, highest lobar CT score). It is therefore likely that a prospective study scoring CT distribution of disease and subsequent guidance of TBB to the lobe with the greatest involvement would yield an even greater association with a positive biopsy.

\section{Relationship between BAL counts and TBB yield}

We found a significantly higher percentage of lymphocytes and a lower percentage of neutrophils in patients with a positive TBB result. Only in 23 cases was the BAL fluid taken from the same lobe as the TBB specimen, but our own data from 40 patients with both upper and lower lobe BAL shows that there is little interlobar variation in the percentage of lymphocytes. ${ }^{15}$ Although one might expect this BAL profile to correlate with a ground-glass pattern on the CT scan, this was not observed.

\section{Relationship between lung function and TBB yield}

Earlier attempts have been made to determine if there is a relationship between the TBB result and pulmonary function parameters and symptom scores. Poe et $a^{3}$ found an association between a positive TBB result and reduced TLCO and increased symptoms, but this was not independent of the chest radiographic stage of the disease. Koontz et al found that a positive TBB result was associated with a restricted FVC $(<80 \%$ predicted) and higher cumulative symptom score, hypothesising that these are associated with more widespread granulomatous involvement. We found no correlation between lung function parameters and presenting symptoms in this study, although the retrospective nature makes it difficult to assess symptom severity confidently. Reduced percentage predicted TLCO was associated with a positive TBB result, but other physiological parameters showed no association. This is likely to reflect the reported association of TLCO with profusion of opacities on the CT $\operatorname{scan}^{16}$ as, on multivariate analysis, percentage predicted TLCO is no longer a significant predictor of a positive TBB result. It has previously been shown that $\mathrm{FEV}_{1}$ correlates with the extent of reticular pattern in sarcoidosis ${ }^{17}$ and, given that both total and lobar reticular scores were associated with a positive $\mathrm{TBB}$, it is surprising that $\mathrm{FEV}_{1}$ did not predict this. However, lung function in this cohort was well preserved compared with that reported by Hansell et al. ${ }^{17}$ 


\section{Demographic features and TBB yield}

A surprising outcome of this study was the strong correlation between a positive TBB result and female gender and a weaker correlation with increasing age which has not been reported previously. While we cannot explain the relationship with gender, it is possible that older patients were less likely to be thought to have sarcoidosis and were therefore more likely to have a biopsy even in the face of a typical scan, and hence a greater chance of a positive TBB yield. This is supported by the fact that the mean age of the cohort is older than our previous studies $^{15}$ and other TBB studies reported. ${ }^{23}$

\section{Study limitations}

As with any retrospective study, there were problems with incomplete data and potential varied clinical practice but, in selecting only those patients who had a CT scan within 6 weeks of bronchoscopy, the association of a positive TBB result with the CT score should be robust. A surprising finding of this study was the low yield of positive TBB for the cohort as a whole. The majority of patients had their CT scan before the diagnostic procedure, with possible selection bias introduced when including only those patients subsequently put forward for TBB. Our cohort could therefore be enriched with patients with more atypical CT features providing an explanation for the low diagnostic yield. Because of the small cohort size, comparison has been made between those with a positive biopsy and those without, the latter group containing those who had either a normal, inconclusive or inadequate biopsy. One could perhaps argue that those with a positive biopsy should be compared with those with a definite negative biopsy (excluding those who were considered inadequate $(n=4)$ or inconclusive $(n=9))$, thus potentially removing any bias from the skill of the bronchoscopist. Such analysis increased the yield in the remaining sample to $61 \%$, but showed that the same associations are significant except for total ground-glass score which becomes $p=0.06$ (from $p=0.04$ ). Considering other factors known to influence yield, the retrospective nature of this study did not allow accurate assessment of total number of biopsies taken and the experience of the bronchoscopist. There was, however, no significant improvement in yield when more than one lobe was sampled.

\section{CONCLUSION}

Patients with a suspected diagnosis of sarcoidosis increasingly undergo high-resolution CT scanning at presentation, both for staging and diagnostic purposes, and many of those patients will have parenchymal disease demonstrated on the CT scan without any visible interstitial change on the chest radiograph. Where histological confirmation is required, TBB is more likely to be positive in those with a greater extent of parenchymal disease on the CT scan, and we recommend that biopsies be taken from the lobe of greatest involvement, paying particular attention to the reticular and ground-glass patterns.

Acknowledgements: The authors thank Wendy Fergusson for statistical advice, Dr Mark O'Carroll for the initial database and Professor John Kolbe for assistance with manuscript review.

Funding: Supported by a grant from the Myrtle Martin Trust.

Competing interests: None.

Ethics approval: The study received approval from the New Zealand Ministry of Health northern regional ethics committee.

\section{REFERENCES}

1. Anon. Statement on sarcoidosis. Joint Statement of the American Thoracic Society (ATS), the European Respiratory Society (ERS) and the World Association of Sarcoidosis and Other Granulomatous Disorders (WASOG). Am J Respir Crit Care Med 1999;160:736-55.

2. Koontz $\mathbf{C H}$, Joyner LR, Nelson RA. Transbronchial lung biopsy via the fiberoptic bronchoscope in sarcoidosis. Ann Intern Med 1976;85:64-6.

3. Poe RH, Israel RH, Utell MJ, et al. Probability of a positive transbronchial lung biopsy result in sarcoidosis. Arch Intern Med 1979;139:761-3.

4. Mitchell DM, Mitchell DN, Collins JV, et al. Transbronchial lung biopsy through fibreoptic bronchoscope in diagnosis of sarcoidosis. BMJ 1980;280:679-81.

5. Koerner SK, Sakowitz AJ, Appelman RI, et al. Transbronchinal lung biopsy for the diagnosis of sarcoidosis. N Engl J Med 1975;293:268-70.

6. Roethe RA, Fuller PB, Byrd RB, et al. Transbronchoscopic lung biopsy in sarcoidosis. Optimal number and sites for diagnosis. Chest 1980;77:400-2

7. Gilman MJ, Wang KP. Transbronchial lung biopsy in sarcoidosis. An approach to determine the optimal number of biopsies. Am Rev Respir Dis 1980;122:721-4.

8. British Thoracic Society Standards of Care Committee. The diagnosis, assessment and treatment of diffuse parenchymal lung disease in adults. Thorax 1999;54:S1-28.

9. Halme M, Piilonen A, Taskinen E. Comparison of endobronchial and transbronchial biopsies with high-resolution CT (HRCIT) in the diagnosis of sarcoidosis. APMIS 2001;109:289-94.

10. Takahashi T, Azuma A, Abe S, et al. Significance of lymphocytosis in bronchoalveolar lavage in suspected ocular sarcoidosis. Eur Respir J 2001;18:515-21.

11. Brauner MW, Grenier P, Mompoint D, et al. Pulmonary sarcoidosis: evaluation with high-resolution CT. Radiology 1989;172:467-71.

12. Muller NL, Kullnig P, Miller RR. The CT findings of pulmonary sarcoidosis: analysis of 25 patients. AJR Am J Roentgenol 1989;152:1179-82.

13. Nishimura K, Itoh $\mathrm{H}$, Kitaichi $\mathrm{M}$, et al. Pulmonary sarcoidosis: correlation of CT and histopathologic findings. Radiology 1993;189:105-9; erratum appears in Radiology 1994;190:907.

14. Winterbauer RH, Lammert J, Selland M, et al. Bronchoalveolar lavage cell populations in the diagnosis of sarcoidosis. Chest 1993;104:352-61.

15. Wilsher ML, Hopkins R, Milne D, et al. Comparison of bronchoalveolar lavage at two sites in sarcoidosis. Respirology 2004;9:98S

16. Muller NL, Mawson JB, Mathieson JR, et al. Sarcoidosis: correlation of extent of disease at CT with clinical, functional, and radiographic findings. Radiology 1989;171:613-8.

17. Hansell DM, Milne DG, Wilsher ML, et al. Pulmonary sarcoidosis: morphologic associations of airflow obstruction at thin-section CT. Radiology 1998;209:697-704. 